\title{
RELACIÓN ENTRE LA HORMIGA Camponotus sp. (Hymenoptera: Formicidae) Y UNA COMUNIDAD DE CACTUS (CACTACEAE) EN EL VALLE DEL RÍO CHILLÓN
} Sidney Novoa S. ${ }^{1}$, Viviana Castro C. ${ }^{2}$, Aldo Ceroni S. ${ }^{2}$ e Inés Redolfi P. ${ }^{1}$

\section{Resumen}

Las observaciones se realizaron en un ecosistema de zona árida en el Valle del Río Chillón, Lima-Perú, el mes de Julio de 2003. En 4 cuadrantes de 15 x 15 m cada uno, se determinó la relación de la hormiga Camponotus sp. con la comunidad de cactáceas. La riqueza de especies de cactus fue $\mathrm{S}=8$ y la hormiga forrajea únicamente en el exterior de los botones florales de la especie Neoraimondia arequipensis ssp. roseiflora. Los resultados sugieren que la hormiga es un bioindicador de nectarios externos en flor al demostrar el éxito de la colecta de alimento líquido en la prueba de presión del gáster. Así mismo, se obtuvo una correlación altamente significativa entre el número de botones florales de $N$. arequipensis y el número de hormigas forrajeando en los botones florales, utilizando el coeficiente de correlación de Pearson. Estas observaciones preliminares del subproyecto dentro del Proyecto Cactáceas de Lima son de gran importancia en el entendimiento de ecosistemas con escasos elementos bióticos y variables abióticas extremas, lo cual sería un interesante aporte para el conocimiento y comprensión de ecosistemas más complejos.

Palabras clave: Cactus, hormiga, interacción, desierto.

\begin{abstract}
Observations were made in an arid ecosystem in the Chillón River Valley, in Lima - Perú, on July 2003. The relation of the cacti community with the Camponotus $\mathrm{sp}$. ant was determined in four 15 x 15 meters quadrants. The richness of the cacti species was $\mathrm{S}=8$ and it was found out that the ant forages only in the outside of the Neoraimondia arequipensis ssp. roseiflora bottom flowers. The results suggest that the ant acts as a bioindicator of the presence of external flower nectarines as the successful gaster pressure test conducted in the collected liquid content indicated. A highly significant correlation using the Pearson coefficient, between the number of bottom flowers in $N$. arequipensis and the foraging ants was obtained. These results on preliminary observations of the Lima's Cactaceae Sub Proyect are very important in this ecosystem with limited biotic elements and extreme abiotic variables. It is an interesting tool for gaining knowledge and for the comprehension of complex ecosystems.
\end{abstract}

Key words: Cacti, ant, interaction, desert.

\section{Introducción}

El Valle del Río Chillón (Departamento de Lima, en las Provincias de Lima y Canta), presenta fondos profundos de zonas llanas cortadas por abruptos costados en quebradas de fuerte pendientes y estrechas gargantas. El clima en las partes media y baja es suave y templado, mientras que en las partes más altas es mas bien frío (ONERN, 1975). En la parte mas baja del piso, la vegetación natural está constituida de cactáceas columnares y otra vegetación reducida. En este sistema, la comunidad de cactus conforma el nivel trófico dominante y es el eje de todos los procesos. En estudios realizados en una de estas especies, Neoraimondia arequipensis ssp. roseiflora (Werd. \& Backbg.) Rauh. Ostolaza et al. (1985), destaca la presencia de negras y grandes hormigas invadiendo los brotes florales, flores abiertas $\mathrm{y}$ frutos. La presencia de hormigas en ecosistemas de zonas áridas debe ser importante objetivo de estudio por las variables puntuales extremas, en un ambiente tan agreste de escasos recursos, en donde la especificidad del nicho ecológico se incrementa. Las hormigas intervienen en el ciclo de nutrientes en la naturaleza, enriquecimiento de los suelos y en una gran diversidad de interacciones tróficas, tanto que se considera que el flujo de energía que pasa a través de ellas es superior al que pasa a través de los vertebrados homotermos que viven en el mismo hábitat (Adlung, 1966; Detrain, 1990; Weseloh, 1996). Esto se debe a que están entre los insectos más altamente sociales por el funcionamiento con sistemas de castas, división de labor, cooperación entre individuos en la cría de los

\footnotetext{
${ }^{1}$ Laboratorio de Control Biológico y Ecología de Artrópodos. Departamento Académico de Biología. Universidad Nacional Agraria La Molina. Av. La Molina s/n Apartado Postal 456. Lima 100. Perú. Dirección electrónica:20000084@lamolina.edu.pe /rhi@lamolina.edu.pe

2 Jardín Botánico “Octavio Velarde Núñez”. Departamento Académico de Biología. Universidad Nacional Agraria La Molina. Av. La Molina s/n Apartado Postal 456. Lima 100. Perú. Correo electrónico: vcastro@lamolina.edu.pe / aceroni@lamolina.edu.pe
} 
jóvenes, empleo de sustancias químicas (feromonas) para comunicarse y a que la reproducción se realiza mediante una casta separada (North, 1996). Las hormigas tienen regímenes alimenticios muy variados, así se las menciona como insectívoras, fitófagas, nectarívoras, granívoras y omnívoras (Retana et al., 1987; Alsina et al., 1988; Dejean et al., 1997) por lo que son consideradas importantes indicadores de los procesos en la dinámica de los ecosistemas. La relación hormiga-cactácea ha sido ya estudiada en otras zonas desérticas del mundo, como la relación entre la hormiga Crematogaster opuntiae Buren en la cactácea Opuntia acanthocarpa Engelmann \& Bigelow en el noreste de Phoenix, Arizona (Pickett \& Clark, 1979), dos especies de hormigas Crematogaster depilis Wheeler e Iridomyrmex pruninosus Royer en Ferocactus cylindraceus (Engelmann) Orcutt (Ruffener \& Clark, 1986; Hunt, 1999). En el Perú, los únicos antecedentes que citan esta interacción son las observaciones de Ostolaza et al. (1985) ya mencionada y de Pizarro (1997) quien indica la visita de hormigas al "ayrampo" Opuntia soehrensii Britton \& Rose, en especial en época de verano, cuando sus frutos maduran.

El presente trabajo, parte inicial de un subproyecto dentro del Proyecto Cactáceas de Lima, tiene por objetivo determinar la relación entre la "hormiga negra" Camponotus sp. con la comunidad de cactáceas en el Valle del Río Chillón, Perú, considerando que este sistema de zona árida, con escasos elementos, podría ser un interesante medio para el conocimiento y comprensión de ecosistemas más complejos.

\section{Materiales y métodos \\ Lugar de investigación}

El área de la investigación fue en el Cerro Umarcata, el cual se encuentra a una distancia de 6 kilómetros de Santa Rosa de Quives, a la altura del kilómetro 69 de la carretera Lima - Canta, a una altitud de 1260 m.s.n.m. a 11'37'39', L.S. y 7646'9', L.O. en el Valle del Río Chillón, Departamento de Lima, en las Provincias de Lima y Canta (Figura 1). La vegetación natural está dominada por comunidades de cactáceas. La temperatura media anual está entre $\operatorname{los} 5^{\circ} \mathrm{C}$ a los $19^{\circ} \mathrm{C}$. La precipitación es prácticamente nula en la parte baja y se hace evidente a partir de los 2000 m.s.n.m., con rango de precipitación de 20 a $850 \mathrm{~mm}$ en promedio anual. La estación de lluvias comprende los meses de Diciembre a Marzo y la estación seca, los meses de Mayo a Setiembre (ONERN, 1975).

\section{Metodología}

Se realizaron dos visitas al lugar: una previa para la colecta de especimenes de la hormiga y observar su comportamiento y la segunda para la toma de datos. En la visita previa se determinó el lugar de forrajeo de la hormiga y se realizó la prueba para comprobar el éxito de la colecta de alimento líquido por la hormiga, para lo cual se presionó suavemente el gáster de 30 individuos según la técnica utilizada por diferentes autores (Cherix, 1981; Retana et al., 1986, 1987; Alsina et al., 1988; Cerdá et al., 1989; Cavia, 1989). Se colectó 20 individuos de la "hormiga negra" en alcohol 70\%, para su posterior determinación en el Museo de Entomología de la UNALM como
Figura 1. Ubicación del área de estudio en el Valle del Río Chillón. 1:100,000 (IGN, 1992).

\section{Camponotus sp.}

En la segunda visita, se trazaron 4 cuadrantes de $15 \times 15$ m cada uno seleccionados aleatoriamente. En el interior de cada cuadrante se determinó la riqueza de especies (S) de cactáceas. En cada individuo de cactus se contabilizó el número de botones florales y el número de individuos de la hormiga Camponotus sp. forrajeando sobre los botones. El muestreo se efectuó en el mes de Julio de 2003 y las observaciones de la presencia de la hormiga entre las 11:00 y 13:00 hs. El trazado de cuadrantes y toma de datos fue realizado por 10 observadores.

Los datos se procesaron en el Laboratorio de Control Biológico y Ecología de Artrópodos y en el Jardín Botánico "Octavio Velarde Nuñez" de la Universidad Nacional Agraria La Molina.

Para el análisis de los datos se calculó el coeficiente de correlación lineal de Pearson entre el número de botones florales y el número de hormigas forrajeando por planta, y se realizó la prueba estadística t-Student (9)gl, en el paquete estadístico Minitab 12.1 para probar la hipótesis de no correlación entre ambas variables $\left(\mathrm{H}_{\mathrm{p}}: \rho=0\right)$. 


\section{Resultados y discusión}

Se determinó una riqueza $\mathrm{S}=8$ de cactus en la zona en estudio, con el mayor número de individuos $(\mathrm{n}=$ 201) pertenecientes a la especie Melocactus peruvianus Vaupel. En esta especie se destaca una estructura diferente llamada "cefalio" en lugar de botones florales. Otras especies también abundantes son Mila nealeana Backeberg y Haageocereus acranthus (Vaupel) Backeberg. Las especies con menor número de individuos fueron: Cleistocactus acanthurus ssp. faustianus (Backeberg) Ostolaza, $H$. pseudomelanostele ssp. aureispinus (Rauh \& Backberg) Ostolaza, Neoraimondia arequipensis ssp. roseiflora (Backeberg) Ritter \& Erort, Opuntia pachypus Schumann y Espostoa melanostele (Vaupel) Borg. (Tabla 1). En esta comunidad, únicamente la especie $N$. arequipensis tuvo presencia de la hormiga, mientras que las otras siete especies no presentaron obreras de Camponotus sp. forrajeando o visitando la planta. Cuatro especies de cactáceas presentaron botones florales: $H$. acranthus $(\mathrm{n}=10), H$. pseudomelanostele $(\mathrm{n}=29)$, Cleistocactus acanthurus $(\mathrm{n}=5)$ y $N$. arequipensis con el mayor número de botones florales $(\mathrm{n}=83)$. Por otro lado, Camponotus sp. $(\mathrm{n}=131)$ se presentó en 67 botones florales de $N$. arequipensis (Tabla 1). La presencia de la hormiga únicamente en $N$. arequipensis en relación a las ocho especies de la comunidad, el alto porcentaje de hormigas visitando a estos cactus cuando forman botones florales, así como su distribución sobre los botones florales (Tablas 2 y 3), puede deberse al hecho que esta especie presenta espacios más amplios y $\sin$ espinas entre las costillas (Figura 3) por los

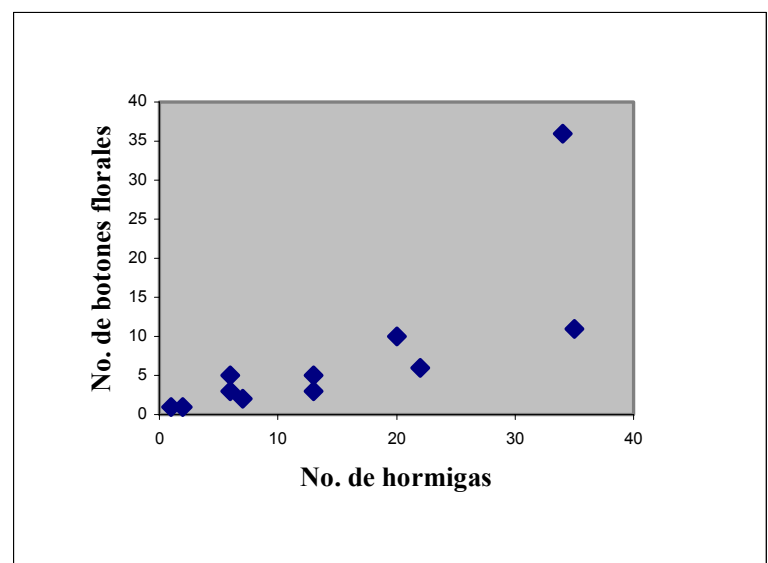

Figura 2. Correlación entre botones florales de Neoraimondia arequipensis ssp. roseiflora y Camponotus sp., en el Valle del Río Chillón: Cerro Umarcata.

cuales pueden desplazarse rápidamente las obreras menores de Camponotus sp., con una longitud del cuerpo de $0.66 \mathrm{~cm}$., que no les permitirían visitar las otras siete especies de cactus, por la gran densidad de espinas que poseen cubriendo inclusive las costillas.
Camponotus sp. forrajeaba únicamente en la zona exterior de los botones florales de $N$. arequipensis en donde permanecía largo tiempo (hasta 20 minutos) y no formaba pistas al desplazarse por las ramas. Por otro lado, la prueba de presión del gáster demostró gran abundancia de alimento líquido transportado por las hormigas, lo que unido a la observación del lugar de forrajeo en la zona externa en los botones florales confirman la relación hormiga-nectarios externos, también observada en México (Pickett \& Clark, 1979; Ruffener \& Clarck, 1986).

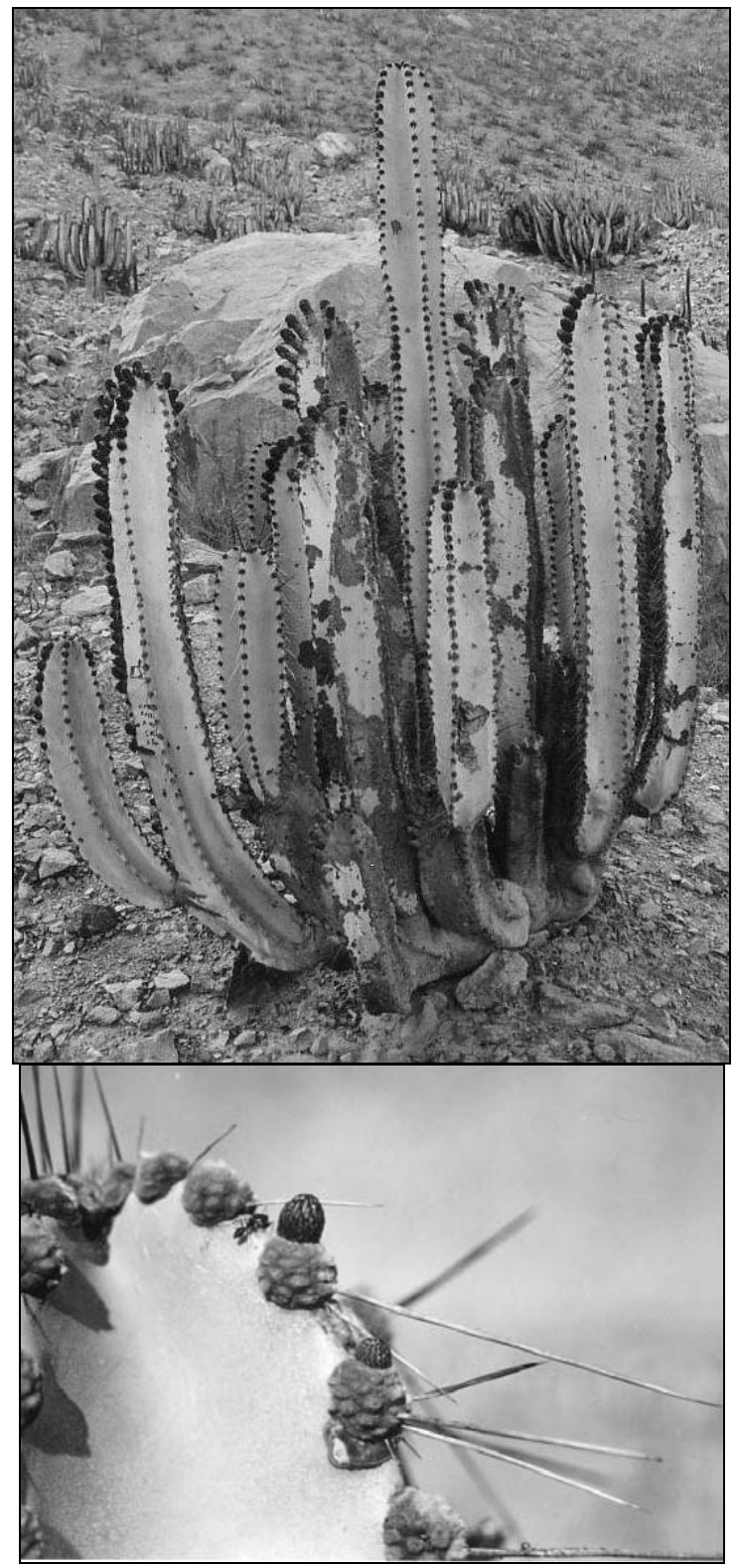

Figura 3. Relación de la hormiga Camponotus sp. (Hymenoptera: Formicidae) en Neoraimondia arequipensis ssp. rosiflora en la comunidad de cactus (Cactaceae) en el Valle del Río Chillón. Fotos: Diego De la Barra (2003). 
Con este resultado se planteó la posibilidad de la existencia de un grado de correspondencia entre la presencia de hormiga y la presencia de botones florales en esta especie de cactácea por lo que se realizó un análisis de correlación entre el número de botones florales por planta y el número de hormigas forrajeando por planta, en los individuos (11 cactus) que alcanzaron la edad adulta, es decir que son capaces de producir botones florales. Dicho análisis arrojó un valor de $\mathrm{r}=0.828\left(\mathrm{t}_{\mathrm{cal}}=4.43,9\right.$ g.l., $\mathrm{P}<$ $0.001)$. Entonces, se concluye que para un $\alpha=0.05$, las evidencias muestrales indican que existe una correlación significativa entre el número de botones por planta y el número de hormigas forrajeando por planta en $N$. arequipensis ssp. roseiflora (Figura 2). Esta relación significativa $(\mathrm{P}<0.001)$ entre Camponotus sp. y $N$. arequipensis es de gran importancia en este ecosistema de zona árida en donde las variables abióticas son extremas y el éxito de la biocenosis depende de finas interacciones entre las variables bióticas. Estas observaciones preliminares permiten elaborar una serie de preguntas que deberán ser absueltas en la continuación del subproyecto iniciado. Así, se esperaría que los hormigueros de Camponotus sp. estén ubicados al pie de los individuos de $N$. arequipensis, lo que contribuiría a un mayor aporte de nutrientes, necesarios para esta cactácea de mayor tamaño y biomasa en relación a las otras siete especies. Igualmente, las flores de $N$. arequipensis serían visitadas por otros artrópodos que conforman una delicada red trófica, posiblemente la única o más importante de este ecosistema.

Tabla 1. Lista de especies, número de individuos, número de botones florales y número de hormigas en las cactáceas del Valle del Río Chillón: Cerro Umarcata.

\begin{tabular}{|c|c|c|c|c|c|}
\hline $\begin{array}{l}\text { Especie de cactáceas } \\
\text { (Anderson, 2001) }\end{array}$ & 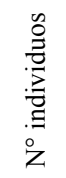 & 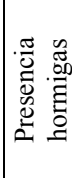 & 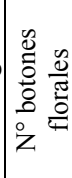 & 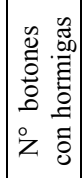 & 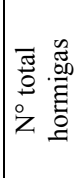 \\
\hline Opuntia pachypus & 17 & no & 0 & 0 & 0 \\
\hline Mila nealeana & 62 & no & 0 & 0 & 0 \\
\hline Haageocereus acranthus & 32 & no & 10 & 0 & 0 \\
\hline $\begin{array}{l}\text { H. pseudomelanostele } \\
\text { spp. aureispinus }\end{array}$ & 57 & no & 29 & 0 & 0 \\
\hline $\begin{array}{l}\text { Cleistocactus acanthurus } \\
\text { spp. faustianus }\end{array}$ & 40 & no & 5 & 0 & 0 \\
\hline Melocactus peruvianus & 201 & no & 0 & 0 & 0 \\
\hline $\begin{array}{l}\text { Neoraimondia } \\
\text { arequipensis spp. } \\
\text { roseiflora }\end{array}$ & 28 & si & 83 & 67 & 131 \\
\hline Espostoa melanostele & 9 & no & 0 & 0 & 0 \\
\hline
\end{tabular}

Tabla 2. Porcentajes de plantas de Neoraimondia arequipensis spp. roseiflora con botones florales, cactus con hormigas y botones florales con hormigas, en el Valle del Río Chillón: Cerro Umarcata.

\begin{tabular}{|c|c|c|}
\hline $\begin{array}{c}\text { Porcentaje de } \\
\text { Plantas con } \\
\text { botones florales }\end{array}$ & $\begin{array}{c}\text { Porcentaje de } \\
\text { plantas con } \\
\text { botones y con } \\
\text { hormigas }\end{array}$ & $\begin{array}{c}\text { Porcentaje de } \\
\text { botones florales } \\
\text { con hormigas }\end{array}$ \\
\hline $32.28 \%$ & $100 \%$ & $80.72 \%$ \\
\hline
\end{tabular}

Tabla 3. Distribución de Camponotus sp. sobre los botones florales de Neoraimondia arequipensis spp. roseiflora, en el Valle del Río Chillón: Cerro Umarcata.

\begin{tabular}{|c|c|c|c|c|}
\hline Media & Mediana & $\begin{array}{c}\text { Desviación } \\
\text { estándar }\end{array}$ & Máximo & Mínimo \\
\hline 1.916 & 2.000 & 1.669 & 8.000 & .0 .000 \\
\hline
\end{tabular}

\section{Conclusiones}

1. La hormiga Camponotus sp. interacciona únicamente con la especie, Neoraimondia arequipensis ssp. roseiflora, de las ocho que conforman la comunidad de cactáceas en el Valle del Río Chillón.

2. Las observaciones de campo y el análisis de datos muestran que existe una relación altamente significativa entre la hormiga Camponotus sp. y la presencia de botones florales de Neoraimondia arequipensis ssp. roseiflora.

3. Camponotus sp. podría ser denominada como un bioindicador de nectarios externos en Neoraimondia arequipensis ssp. roseiflora, por lo que se continuarán los estudios en la anatomía de la flor.

4. Estudios sobre la fenología de esta planta podrían descartar la mayor época de floración y por lo tanto el aumento de los visitantes florales como Camponotus sp.

\section{Agradecimientos}

Los autores expresan su agradecimiento a la Dra. Consuelo Arellano Ugarte, por la revisión del manuscrito y sugerencias en la presentación del análisis estadístico. A la Dra. Cecilia Díaz-Castelazo, del Departamento de Ecología Vegetal, Instituto de Ecología de Veracruz, México y al Dr. Carlos Ostolaza, Presidente de la Sociedad Peruana de Cactus y Suculentas "SPECS" por facilitar bibliografía sobre hormigas-cactáceas. A la Bachiller Silvia Castro por la determinación del género Camponotus. A los estudiantes de Biología de la UNALM que colaboraron en la toma de datos. Esta investigación 
fue financiada por el FEDU (Fondo Investigación Universitaria) de la Universidad Nacional Agraria La Molina.

\section{Literatura citada}

Anderson E.F. 2001. The Cactus Family. Timber press.

Adlung K.G. 1966. A critical evaluation of the european research on use of red wood ants (Formica rufa group) for the protection of forests against harmful insects. Dpto. Agricultural Research Service. París. 57: 167-189.

Alsina A., Cerda X., Retana J. \& Bosch J. 1988. Foraging ecology of the aphid-tending ant Camponotus cruentatus (Hymenoptera, Formicidae) in a savanna-like grassland. Misc. Zool. 12: 195-204.

Cavia V. 1989. Régimen alimenticio de la hormiga Formica subrufa (Hymenoptera: Formicidae). Ses. Entomol. ICHN-SCL. VI: 97-107.

Cerda X., Retana J., Bosch J. \& Alsina A. 1989. Exploitation of food resources by the ant Tapinoma nigerrimum (Hym. Formicidae). Acta Oecologica. 10(4): 419-429.

Cherix D. 1981. Contribution á la biologie et á l'écologie de Formica lugubris. Le probléme des super-colonies. Thesis, Lausanne.

Dejean A., Ngnegueu P.R., Durand J.L. \& Bourgoin T. 1997. The influence of ants (Hymenoptera: Formicidae), particularly tramp species, on the proliferation of a maize pest. Sociobiology. 30(1): 85-93.

Detrain C. 1990. Field study on foraging by the polymorphic ant species, Pheidole pallidula. Ins. Soc. 37 (4): 315-332.
Hunt C. 1999. CITES. Cactaceae Checklist. ${ }^{\text {nd }}$. Ed. Instituto Geográfico Nacional. 1992. Carta Nacional 1:100000. Hoja 24-j. Lima, Perú.

North R. 1996. Ants. World Wildlife Series. London.

Oficina Nacional de Evaluación de Recursos Naturales. 1975. Informe Técnico del Proyecto de Irrigación Marcapomacocha, Lima. Perú.

Ostolaza C., Mitich W.L. \& King J.M. 1985. Neoraimondia arequipensis var. roseiflora (Werd \& Backbg) Rauh. Cactus and Succulent. Journal (U.S). 57: 60-64.

Pickett C.H. \& Clark W.D. 1979. The function of extrafloral nectarines in Opuntia acanthocarpa (Cactaceae). American Journal of Botany. 66: 618625.

Pizarro J.O. 1997. Cactus y animales: Simbiosis maravillosa. Quepo. 11: 21-28.

Retana J., Bosch A., Alsina A. \& Cerda X. 1987. Foraging ecology of the nectarivorous ant Camponotus foreli (Hymenoptera, Formicidae) in a savanna-like grassland. Misc. Zool. : 187-193.

Retana J., Cerda X., Alsina A. \& Bosch J. 1986. Importancia del alimento sólido y del alimento líquido en el régimen trófico de la hormiga Cataglyphis cursor (Formicidae). Ses. Entom. ICHN-SCL.4:136-146.

Ruffener G.A. \& Clark W.D. 1986. Extrafloral nectar in Ferocactus acanthodes (Cactaceae): composition and its importance to ants. American Journal of Botany. 73: 185-189.

Weseloh R.M. 1996. Effect of suplemental foods on foraging behavior of forest ants in Connecticut. Env. Ent. 25 (4): 848-853. 